\title{
Applying Intermittent Energy Distribution for Evading Energy Holes in Wireless Sensor Network
}

\author{
ArpithaVasudev, A.M. Sowmya and G. Manjula
}

\begin{abstract}
This paper proposes a non-uniform answer for the vitality opening issue. The system is shaped by a huge number of modest sensors, arbitrarily scattered over a roundabout region and the sink situated at the inside. The sensors deliver information with the same rate and the information makes a trip from the hubs to the sink in a multibounce form, utilizing a most limited way steering. The essential thought, so as to stay away from the vitality gap around the sink, is to prepare each organize zone with such a great amount of vitality as the movement heap of that zone. Firstly, an unadulterated systematic expression for the activity load is determined and after that, taking into account this expression, a non-uniform organization is proposed, with variable surface thickness, for a specific number of lethargic hubs. Every time a dynamic hub depletes its battery, a close torpid hub gets to be dynamic and has its spot.
\end{abstract}

Index Words--- Wireless Sensor Networks, Energy Holes, Intermittent Method.

\section{INTRODUCTION}

$\mathrm{W}$ IRELESS sensor systems (WSN) remain a thriving exploration field for over 10 years now. They typically comprise of countless cost secluded sensors which are sent, haphazardly or intentionally, terminated a topographical zone. The goal of such a system is to sagacityvarious sort of surrounding capricious and report this data to a social affair theme (additionally called sink). The dispersal of information is expert by a remote callous. Hence the WSNs are a great decision for situations everywhere the foundation of reinforced systems is inconceivable or exceptionally hard to accomplish. There arenumerous varieties of such systems relying upon the tender prerequisites and ecological requirements. Broad-spectrum prologue to the meadow can be initiate in [1].

The remote way of WSNs styles it conceivable to develop expansive rule systems, casing completely a topographical zone. The most straightforward approach to accomplish this is by a multi-jump form, i.e., feelers createinformation as well as in the meantime demonstration additionally as transfers for exchanging those information detected by nearby sensors.A key variable of this kind of systems is the vitality utilization, since it is firmly identified with the system's lifetime when

ArpithaVasudev, Lecturer, Dept. of CSE, Bangalore College of Engineering and Technology, VTU, Bengaluru, India. E-mail:arpitha.dev9@gmail.com

A.M. Sowmya, Dept. of CSE, SriSai Ram College of Engineering, VTU, Bengaluru, India.E-mail:sowmyabarathi@gmail.com

G. Manjula, Assistant Professor, Dept. of CSE, SriSai Ram College of Engineering, VTU, Bengaluru, India.E-mail:sowmyabarathi@gmail.com DOI:10.9756/BIJSESC.8281 antennae are cordless hang on as it is for the most part the case in WSNs.

Despite the fact that there are various methodologies endeavoring to diminished the expended vitality [2], the supposed vitality gap has been turned out to be an unavoidable issue on account of a uniform system with a static sink [3], [4]. This issue emerges at the point when the voyaging information approaches the sink. Because of merging, the sensors close to the sink convey the weight of sending more information than whatever is left of the hubs and therefore the region around the sink is rapidly left purge of vitality. This paper is attempting to go up against the vitality gap issue with non-uniform vitality appropriation.

As Lian et al. portrayed in [5], to stay away from the vitality opening, the regions close the sink could be furnished with torpid hubs, as vitality stores. At the point when a dynamic hub loses its vitality, a torpid hub has its spot and constant the past typical operation. In any case, they just give the arrangement spellbindingly what's more, examine the general structure, the viability and the attainability, deprived of giving the correct integer of the required torpid hubs, nor the required arrangement thickness. Moreover, they are not worriedabout theprocedure of exchanging between the depletedand the new hub. The slog exhibitedhere utilizationsthe matching primary thought as in [5] yet gives an explanatory countenance to the hub's thickness as a capacity of the separation from the drop, the real numeral of the prerequisite torpid hubs and a straightforward calculation for the exchanging progression between the lethargic dynamic countries.

\section{RELATED WORK}

Different non-uniform answers for the vitality gap issue have stood anticipated. The easiest one is the non-uniform hub sending, as indicated by which the apparent thickness of pivots is capricious, resilient further hubs on the zones close to the sink. These additional hubs are detecting and handing-off information as the typical ones do. In the fundamentally the same as arrangement of non-uniform vitality, the distinction is that the additional hubs are really lethargic, sitting tight for a foreigner hub to fumes its succession until they make a move. Together the above arrangements have been proposed by the same creators in [5]. This paper broadens the later slant by determining an equation for the thickness of the lethargic hubs. Wu et al. in [4] are taking after the main line of attackalong with a unique directing convention which adjusts the movement stack among hubs with the same separation from the sink. The disadvantage is that the organization is not arbitrary but rather needs extremely cautious situating of hubs and 
moreover, it needs a tremendous number of additional hubs (the quantity of hubs takes after a arithmeticalextent whereas drawing closer the sink).

Ammari and Das in [7] intend a blended plan, taking into account concentrictrinkets nearbythe sink, with variable starting freestyle glassyand inconstant radio-run for every ring. There are numerous different arrangements in view of variable radio-range, for example, [8], [3] furthermore, [9]. Alternative group of non-uniform arrangements depends on the blend of sole and multibounce spread. The hubs close to the sink convey a piece of their information by amulti legway, whereas whatever remains of the information are conveyed specifically to the sink by a solitary jump. In actuality, hubs that are not near the sink take after the typical multi-bounce worldview, conveying most or the majority of their information to their neighbors. This kind of arrangement is displayed without precedent for [10] and it have been trailed by numerous variations, for example, [11] and [12]. The expository equation for the hub's thickness gritty later in this work is enlivened by [13].

The creators depict a scientific display for the vitality opening, expecting a undeviating and thick system. They isolate the system territory hooked on concentric rings and gauge the movement heap of every ring as a capacity of the ring number. The drudgery introduced here amplifies the ring demonstrate by utilizing a constant variable tactic and subsequently the inferred recipe for the activity stack does not encompass the specific concentricring number, yet just the separation from the sink. The proposed capacity of the torpid hubs thickness that takes after depends on this explanatory articulation of the activity stack.

\section{EXISTING SYSTEM}

Extend $\mathrm{d}$ assumes an essential part in WSNssince by evolving its esteem, the normal level of the system likewise changes. The point here isto determine a nearby connection flanked by the normal notch $g$ and the range $d$ as takes after. Prior to the hubs' sending, an arbitrary point $\mathrm{P}$ is picked and afterward, N01 hubs are arbitrarily spread over the system zone lastly, the last hub being set at point P. The N01 hubs are consistently dispersed over the system zone with a steady surface thickness $0=\mathrm{A}$, so there are (by and large) d2 hubs inside the scope of point $\mathrm{P}$. Take note of this expect either thatpoint $\mathrm{P}$ is not at the system limits or unending system measurements (a broad talk of this outskirt impact can be found in). This work embraces the equivalentdefinition for the heap asalso.

Beneath the conditions portrayed in, the vitality consumption is just identified with the information conveyed by every hub. The information of a hub $\mathrm{v}$ incorporates not just the bundles created by the hub itself additionally those bundles created by the relatives of $\mathrm{v}$ in the steering tree (hub $\mathrm{v}$ goes about as a hand-off for these hubs). The movement stacks of a hub are the number of parcels prepared to bediffused at hub $\mathrm{v}$ at a specific time case. Given that all hubs deliver one parcel for each time unit, the total number of bundles at any hub adds up to the quantity of its relatives in addition to the one bundle delivered by the hub itself and obviously, N0. Clearly the vitality utilization is relative to the movement stack (basically stack in the future). Since the heap of a particular hub is an irregular variable, the normal heap of a range is characterized as the mean estimation of the heaps of all hubs inside this range.

A strict demarcationfor the normal heap of a specific fact (not a whole range) can be characterized as takes after. Assume that every purpose of the system has polardirections. The heap of a hub $\mathrm{v}$ isa detached arbitrary inconstant which relies on upon the attributes of the organize N0, g, the steering arrangement and the directions of that hub. For an altered blend of N0, g and steering, the likelihood of a hub $v$ to have stack ' $v$ will be connected as it were with the directions of that hub. Let indicates the likelihood mass capacity of the irregular variable gives the likelihood of a hub with directions to have stack consider the $r$ as ceaseless parameters of the likelihood bulk capacity.

\section{PROPOSED SYSTEM}

After the arrangement the dynamic hubs frame a directing tree, taking after the typical way, i.e., the sink starts the steering pyramid development itself existence the root hub and whatever is left of the hubs begin conveying information to the sink. Amid the typical maneuver of the system the torpid hubs are just occasionally earshot for an INVITATION motion from the dynamic hubs. The control motions amongst dynamic and torpid hubs might be exchange through an alternate channel than the standard (or information) one, with a specific end goal to maintain a strategic distance from any random gatherings from the torpid hubs and in this way, to keep their battery as crisp as could reasonably be expected.

Upon the gathering of an INVITATION flag, a lethargic hub reacts to the despatcher with a READY flag, alongside its ID. At the point when a dynamic hub is near fumes its battery, sends an INVITATION flag and sits tight for the READY reacts. It picks the react that originates from the closer lethargic hub and after that conveys to this hub a WAKE-UP flag. In the meantime the depleted hub illuminates the sink for its pending demise by sending a NEWTOPOLOGY flag through the standard channel. A lethargic hub gets to be a dynamic one when it gets a WAKE-UP flag. It opens its circuit and holds up the control memosfrom the sink to take an interest in the development of the following directing tree. The sink, quickly in the wake of getting the NEW-TOPOLOGY flag, starts the development of another directing tree. The coming about tree will contain the previous lethargic hub, yet as a dynamic hub now, with a crisp battery. After that, the depleted hub pull back from; the system and shuts its circuit.

\section{A. Algorithm: The active Swapping algorithm}

\section{Algorithm node}

Step 1: If State=Active

Step2: State=Active or Sleeping

Step3: If Active, Receive data and send data to next node 
Step4: On exhausting the battery,send invitation to all nodes

Step5: Once sent receive the node ID of ready nodes

Step6: Collect node details and wake up nodes, then Get the new topology and send it to sink

Step7: When battery is almost zero,Use the new topology for data transfer

The above algorithm demonstrates the procedure of the adjustment between a dynamic and a torpid hub, in pseudo code. See here that there are no contrasts amongsta dynamicand a torpid hub, aside from their underlying estimation of themunicipal capricious. Both sorts of hubs are running the same calculation and are really the unchanged, in this manner, the best possible state estimation of every sensor can be decided before sending.

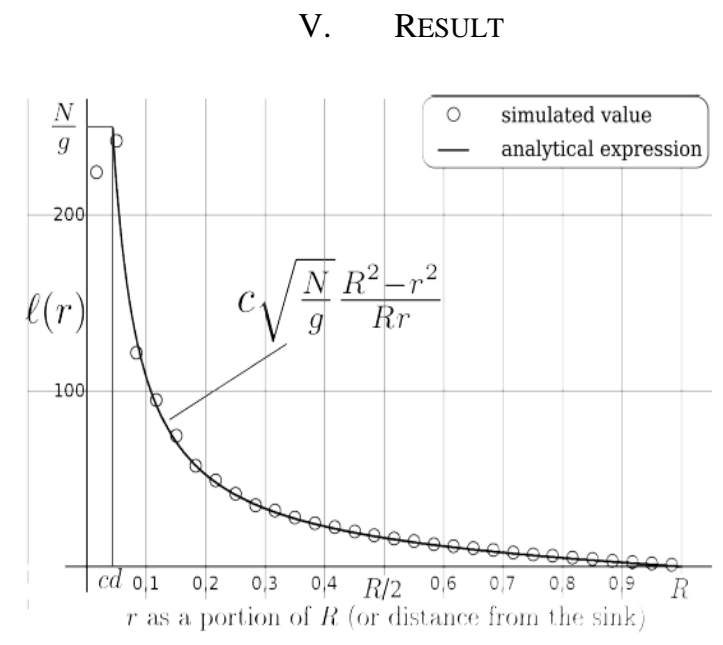

Figure 1: General Vitality Consumption

The general vitality consumption Figure 1 is just because of the information exchange. Another key variable, separated from the epoch, of any non-uniform arrangement is the vitality usage, signified by $u$. This is the rate of the utilized vitality as a small amount of the aggregate beginning vitality and obviously the bigger the u the lesser the waste vitality. All reenactments are performed for asettle number of dynamic hubs.

\section{CONCLUSION}

Above technique gives an solution of the vitality gap issue utilizing torpid hubs with the same surface thickness as the activity stack. Initial, an expository articulation of the activity stack is inferred and after that an arrangement procedure, in view of this expression, is created alongside a straightforward calculation for the exchanging between the dynamic lethargic states. The number of required lethargic hubs is not irrelevant, around 10 times. The underlying dynamic ones, however the remuneration is a change of thesystem lifetime around 50 times. Given that the cost of the remote sensors must be little, the proposed arrangement is considered as moderate as for the hubs cost.

\section{REFERENCES}

[1] T.Y. Wu and C.H. Lin, "Low-SAR path discovery by particle swarm optimization algorithm in wireless body area networks", IEEE Sensors Journal, Vol. 15, No. 2, Pp. 928-936, 2015.

[2] C. Yi, L. Wang and Y. Li, "Energy efficient transmission approach for WBAN based on threshold distance", IEEE Sensors Journal, Vol. 15, No. 9, Pp. 5133-5141, 2015.

[3] J. He, Y. Geng, Y. Wan, S. Li and K. Pahlavan, "A cyber physical testbed for virtualization of RF access environment for body sensor network”, IEEE Sensors Journal, Vol. 13, No. 10, Pp. 3826-3836, 2013.

[4] D. Liu, Y. Geng, G. Liu, M. Zhou and K. Pahlavan, "WBANs-Spa: An energy efficient relay algorithm for wireless capsule endoscopy", Pp. 1-5, 2015.

[5] D. He, S. Chan and S. Tang, "A novel and lightweight system to secure wireless medical sensor networks", IEEE journal of biomedical and health informatics, Vol. 18, No. 1, Pp. 316-326, 2014.

[6] S. Movassaghi, M. Abolhasan, J. Lipman, D. Smith and A. Jamalipour, "Wireless body area networks: A survey", IEEE Communications Surveys \& Tutorials, Vol. 16, No. 3, Pp. 1658-1686, 2014.

[7] M. Li, W. Lou and K. Ren, "Data security and privacy in wireless body area networks," IEEE Wireless Communications, Vol. 17, No. 1, Pp. 51-58, 2010.

[8] C. Hu, F. Zhang, X. Cheng, X. Liao and D. Chen, "Securing communications between external users and wireless body area networks", Pp. 31-35, 2013.

[9] J. Lai, R. H. Deng, C. Guan and J. Weng, "Attribute-based encryption with verifiable outsourced decryption", IEEE Transactions on information forensics and security, Vol. 8, No. 8, Pp. 1343-1354, 2013.

[10] R. Lu, X. Lin and X. Shen, "SPOC: A secure and privacy-preserving opportunistic computing framework for mobile-healthcare emergency", IEEE Transactions on Parallel and Distributed Systems, Vol. 24, No. 3, Pp. 614-624, 2013.

[11] H. Zhao, J. Qin and J. Hu, "An energy efficient key management scheme for body sensor networks," IEEE Transactions on Parallel and Distributed Systems, Vol. 24, No. 11, Pp. 2202-2210, 2013.

[12] D. He, S. Chan, Y. Zhang and H. Yang, "Lightweight and confidential data discovery and dissemination for wireless body area networks", IEEE journal of biomedical and health informatics, Vol. 18, No. 2, Pp. 440-448, 2014.

[13] C.C. Tan, H. Wang, S. Zhong and Q. Li, "IBE-Lite: A lightweight identity-based cryptography for body sensor networks", IEEE journal of biomedical and health informatics, Vol. 13, No. 6, Pp. 926-932, 2009.

[14] D. Boneh and M. Franklin, "Identity-based encryption from the Weil pairing", SIAM journal on computing, Vol. 32, No. 3, Pp. 586-615, 2003.

[15] J. Liu, Z. Zhang, X. Chen, and K. S. Kwak, "Certificateless remote anonymous authentication schemes for wireless body area networks," IEEE Transactions on Parallel and Distributed Systems, Vol. 25, No. 2, Pp. 332-342, 2014. 